\title{
A influência do método Pilates no desenvolvimento motor de crianças: um estudo de intervenção
}

http://dx.doi.org/10.11606/1807-5509202000020249

\author{
Mariele da Silva HERNANDEZ \\ Simone LARA* \\ J aqueline Beck RODRIGUES* \\ Rodrigo de Souza BALK* \\ Susane GRAUP*
}

*Universidade

Federal do Pampa,

Uruguaiana, RS, Brasil.

\section{Resumo}

0 Desenvolvimento motor consiste em uma mudança contínua do comportamento motor ao longo da vida, provocada pela interação entre a exigência da tarefa motora, biologia do indivíduo e condições do ambiente. Neste sentido, o objetivo deste estudo foi analisar a influência do Método Pilates sobre o desenvolvimento motor de crianças e verificar se o desenvolvimento motor está associado ao estado nutricional e ao sexo das mesmas. A amostra foi composta por 58 alunos com idade de 7 anos, sendo 55,2\% do sexo feminino. 0 estudo é caracterizado como quase-experimental, composto por pré-teste e pós-teste e um tratamento experimental com o Método Pilates. 0 Desenvolvimento Motor foi avaliado por meio da bateria de testes Movement Assessment Battery for Children - MBAC, divididos em três categorias: destreza manual, habilidades com bola e equilíbrio. Para verificar o estado nutricional foi utilizada uma balança digital e um estadiômetro. Os resultados mostraram que o desenvolvimento motor teve uma melhora significativa após a aplicação do método Pilates. Não houve diferença estatística entre os sexos e associação entre o estado nutricional e o desenvolvimento das habilidades motoras. Com base nos resultados é possivel concluir que o Método Pilates pode contribuir com o desenvolvimento motor de crianças.

Palavras-chave: Desenvolvimento Infantil; Equilibrio Postural; Atividade Motora; Criança.

\section{Introdução}

O desenvolvimento motor consiste em uma mudança contínua do comportamento motor ao longo da vida, provocada pela interação entre a exigência da tarefa motora, biologia do indivíduo e condiçóes do ambiente ${ }^{1}$. Importantes relaçóes entre o desenvolvimento infantil e fatores ambientais têm sido reportadas, muitas vezes de forma mais expressiva que a própria vulnerabilidade biológica da criança, sugerindo que o ambiente é capaz de modular os riscos a que as crianças estão expostas ${ }^{2}$.

Considerando o fator ambiental, a literatura aponta que a participação regular em atividades esportivas e ginásticas apresentam maior evolução do desenvolvimento de habilidades motoras na infância ${ }^{3}$. Sendo assim, as atividades físicas são de grande importância biológica, psicológica, social e cultural, pois por meio da execução dos movimentos, as crianças interagem com o meio ambiente, relacionam-se com os outros, aprendem sobre si, sobre seus limites e sua capacidade de solucionar os problemas, proporcionando as vivencias para garantir a aquisição de habilidades motoras ${ }^{4}$. Desta forma, as atividades vivenciadas, representam um importante caminho para o desenvolvimento integral da criança, referentes aos domínios relacionados ao seu conhecimento corporal 5 .

Contudo, apesar da importância das crianças praticarem atividades físicas, uma das principais oportunidades de acesso destas às práticas regulares e orientadas é a Educação Física Escolar ${ }^{6}$, o que se constitui, em muitos casos, numa lacuna, pois faltam professores capacitados nos primeiros anos do Ensino Fundamental para atender a este público 
dentro das aulas de Educação Física ${ }^{7}$. Esse fator contribui negativamente sobre o desenvolvimento motor das crianças, pois a infância representa a fase em que os principais parâmetros de habilidades motoras atingem seu pico de desenvolvimento ${ }^{1}$.

Dentre as modalidades de exercício físico que podem ser abordadas na infância, a fim de melhorar o desenvolvimento motor das crianças, destaca-se o Método Pilates, que consiste em uma técnica de controle consciente de todos os movimentos musculares do corpo $^{8}$. Conforme Pereira, Junior e Campos ${ }^{9}$, o conceito do método se dá a partir de seis princípios básicos e fundamentais: centro (ponto principal do método, "núcleo do corpo"), concentração (importante na realização dos exercícios, "a mente guia o corpo"), controle (consciência de todos os movimentos ao se exercitar), respiração (realizada em todos os movimentos, com ritmo) e por último a fluidez (depois de se adquirir coordenação dos movimentos,

\section{Método}

Trata-se de uma pesquisa quase-experimental (sem grupo controle). Foram incluídos estudantes do $1^{\circ}$ ano do Ensino Fundamental, de uma escola pública do município de Uruguaiana, interior do Rio Grande do Sul, Brasil. Para a composição da amostra foram utilizados os seguintes critérios de inclusão: a) apresentar o termo de consentimento livre esclarecido assinado pelos pais ou responsáveis, b) estar matriculado no $1^{\circ}$ ano do ensino fundamental, independente do sexo e idade. Os alunos que foram excluídos da amostra foram aqueles que apresentavam deficiências físicas, visuais e/ou cognitivas atestado por meio de laudo médico.

Para a execução do projeto, primeiramente realizouse uma reunião com os pais para explicar os objetivos e o funcionamento do projeto, bem como, para expor os benefícios que os estudantes poderiam ter com a prática de atividades físicas regulares. Foram entregues os termos de consentimento livre e esclarecido para os responsáveis assinarem, informando que o assentimento do menor seria considerado. O estudo foi conduzido dentro dos padrốes exigidos pela Declaração de Helsinque, aprovado pelo Comitê de Ética e Pesquisa da universidade local, sob parecer $n^{\circ}$ 457.088.

O protocolo de avaliaçấo do estudo consistiu em:

- Avaliaçáo do desenvolvimento motor: por meio da Bateria para Avaliaçáo do Movimento de Crianças (Moviment Assessment Battery for Children-Moviment com a prática, desenvolver-se-á um ritmo, tornando o exercício contínuo). Neste contexto, a utilização do Método Pilates na infância tem apresentado influência positiva sobre o desenvolvimento motor, sendo que no estudo de Montanez e LARA ${ }^{10} \mathrm{com}$ crianças de seis a oito ano de idade, foi possível identificar melhoras significativas na motricidade fina, equilíbrio, esquema corporal, organização espacial e quociente motor geral após 28 sessôes de Pilates solo.

Apesar do crescimento do número de publicações envolvendo o método Pilates em diferentes contextos e populaçóes, sua abordagem, em crianças saudáveis no contexto escolar, é limitada. Dessa forma, o presente estudo objetivou identificar a influência do método Pilates sobre o desenvolvimento motor de escolares do $1^{\circ}$ ano do Ensino Fundamental, e identificar possíveis associaçóes entre o desenvolvimento motor, o estado nutricional e o sexo das crianças avaliadas.

$A B C$ 2), que consiste em uma lista checagem e um teste de desempenho motor aplicável em clínicas e escolas para detectar dificuldades motoras em crianças de 4 a 16 anos $^{11}$, validada para a população brasileira por Valentini, Ramalho e Oliveira ${ }^{12}$. O protocolo utilizado foi a faixa de idade 2 ( 7 a 10 anos), sendo os testes divididos em três categorias: teste de destreza manual, testes de habilidades com bola e testes de equilíbrio. O teste de destreza manual é composto pelas avaliaçóes de colocar os pinos, passar o cordão e trilha da bicicleta. O teste de habilidades com a bola é comporto por: lançar a bola e receber com uma das mãos e lançar o saco de feijão. E o teste de equilíbrio tem as avaliaçóes de equilíbrio sobre uma prancha, andando sobre a linha e saltando com um pé só no tapete.

Para classificar o desenvolvimento motor, foram utilizadas três categorias: zona vermelha (encontra-se abaixo do percentil 5 , e indica dificuldade de movimentação significante), zona laranja (entre o $5^{\circ}$ percentil ao $15^{\circ}$ percentil, e representa risco de dificuldade motora) e zona verde (acima do percentil 16 e indica desenvolvimento típico).

- Avaliaçáo do estado nutricional: Foi utilizado uma balança digital e um estadiômetro fixado na parede. $\mathrm{O}$ cálculo para a definição do Índice de Massa Corporal - IMC foi massa corporal/estatura ao quadrado e para a classificação foi utilizada a tabela do Projeto 
Esporte Brasil (PROESP-Br). As avaliaçōes foram feitas individualmente na sala de ginástica da escola, onde foram montadas as estruturas que cada teste exigia, estando o ambiente em silêncio para a concentração do indivíduo, tendo duraçáo de aproximadamente 30 minutos cada.

O protocolo de intervenção consistiu em três grupos de exercícios do Método Pilates (um inicial, outro intermediário e outro avançado, conforme quadro 1), com enfoque em todos os princípios do Método. As sessōes foram divididas em três momentos: aquecimento, parte principal e relaxamento. No aquecimento foram utilizados exercícios básicos, desenvolvendo atividades para o trabalho da respiração com duração de 5 minutos. Na parte principal, os alunos realizaram atividades no solo (MatPilates), com acessórios (bosu, bola suíça, arco mágico e meia lua) e com aparelhos (Cadillac, Reformer, Barriel, Chair). No relaxamento, foram utilizadas atividades de volta à calma com músicas relaxantes ( 5 minutos). As sessōes foram realizadas uma vez por semana, com duração aproximada de 50 minutos cada, durante 5 meses.

Para a aplicação do protocolo de exercícios do Método Pilates, as crianças foram subdivididas em pequenos grupos, a fim de garantir que todos os princípios fossem aplicados, sendo que os exercícios eram supervisionados por acadêmicos previamente treinados.

QUADRO 1- Protocolo dos exercícios do Método Pilates.

\begin{tabular}{|l|l|l|}
\hline Protocolo & Aparelhos & MAT e acessórios \\
\hline Protocolo 1 & $\begin{array}{l}\text { - Foot work, Arms: pulling- Reformer } \\
\text { - Spine Stretch, Tower - Cadillac } \\
\text { - Horse, Leg extension - Barrel } \\
\text { - Horse Back, Swan front - Chair }\end{array}$ & $\begin{array}{l}\text { - Leg circles, Scissors, Body up and down, } \\
\text { Body extension (Meialua) } \\
\text { - Rollup, exercício de Ponte, Legpull } \\
\text { front, Swan (Bozu) }\end{array}$ \\
\hline Protocolo 2 & $\begin{array}{l}\text { - Stomach massage, Arms: up and down - Reformer } \\
\text { - Spinestretch: variação em pé, Mermaid - Cadillac } \\
\text { - Legextension, horse: variação ajoelhado - Barrel } \\
\text { - Exercício de Ponte, Pumponleg front - Chair }\end{array}$ & $\begin{array}{l}\text { - Knee extension, Onde leg up and down } \\
\text { (Meia lua) } \\
\text { - Exercício de Ponte unipodal, Legpull } \\
\text { front unipodal (Bozu) }\end{array}$ \\
\hline Protocolo 3 & $\begin{array}{l}\text { - Leg lowers, Leg extension - Reformer } \\
\text { - Sit up, Monkey - Cadillac } \\
- \text { Mermaid, Horse: variação com Magic circle - Barrel } \\
\text { - Hamstring stretch, Ponte unipodal - Chair }\end{array}$ & $\begin{array}{l}\text { Ponte, Spine Twist, Single leg lifting, } \\
\text { Swan (bola suíça) }\end{array}$ \\
\hline
\end{tabular}

Inicialmente foram avaliadas 110 crianças, correspondentes ao número total de alunos matriculados no $1^{\circ}$ ano. Porém, os pesquisadores consideraram uma frequência mínima de $75 \%$ de participação com o método Pilates, e com isto, a amostra final foi composta por 58 crianças, sendo 32 do sexo feminino.

Para a análise dos dados foi utilizada a estatística descritiva por meio de valores de mediana, intervalo interquartil e frequências. Para avaliar a normalidade das variáveis foi feito o teste de Kolmogorov-Smirnov, que indicou uma distribuição não normal. Desta forma, para analisar a diferença entre os testes foi utilizada o teste Wilcoxon, enquanto para analisar a diferença entre os sexos foi utilizado o teste " $U$ " de Mann-Whitney. Para identificar a associação das variáveis foi utilizado o teste Qui-quadrado. $\mathrm{O}$ tamanho do efeito entre as intervençóes foi avaliado pelo Teste de Cohen (d) e a Mínima Diferença Detectável foi avaliada pela equação $M M D=z$ escore do nível de confiança*desvio padrão baseline ${ }^{*}\left(2\left[1-\mathrm{r}_{\text {teste-reteste }}\right]\right)^{13}$. Para todas as análises foi considerado um nível de significância de $0,05 \quad(\mathrm{z}=1,96)$. 


\section{Resultados}

Foram avaliados 58 alunos, sendo $55,2 \%$ do sexo feminino, todos com 7 anos de idade. A descrição das frequências das variáveis analisadas está apresentada na TABELA 1, sendo possível observar que a prevalência de déficits motores foi de $67,3 \%$ no pré-teste e $29,3 \%$ no pós-teste. Em relação ao estado nutricional, os valores de excesso de peso (sobrepeso + obesidade) atingiram mais de $35 \%$ da amostra em ambos os testes.

TABELA 1- Distribuição da frequência pré e pós experimento das variáveis analisadas, Uruguaiana, 2015.

$\mathrm{N}=$ amostra, $\%=$ percentual, $\mathrm{IC}=$ intervalo de confiança.

\begin{tabular}{|c|c|c|c|c|}
\hline \multirow{2}{*}{ Variável } & \multicolumn{2}{|c|}{ Pré } & \multicolumn{2}{|c|}{ Pós } \\
\hline & $\mathbf{N}$ & $\%$ (IC) & $\mathbf{N}$ & $\%(\mathrm{IC})$ \\
\hline \multicolumn{5}{|l|}{ Sexo } \\
\hline Masculino & 26 & $44,8(25,7-63,9)$ & - & - \\
\hline Feminino & 32 & $55,2(38,0-72,4)$ & - & - \\
\hline \multicolumn{5}{|c|}{ Desenvolvimento motor } \\
\hline Zona verde & 1 & $1,7(0,23-3,17)$ & 30 & $51,7(33,8-69,6)$ \\
\hline Zona laranja & 18 & $31,0(0,96-52,3)$ & 11 & $19,0(0,42-42,2)$ \\
\hline Zona vermelha & 39 & $67,3(54,6-82,0)$ & 17 & $29,3(7,66-50,9)$ \\
\hline \multicolumn{5}{|l|}{ IMC } \\
\hline Normal & 33 & $56,9(40,0-73,8)$ & 37 & $63,8(48,3-79,3)$ \\
\hline Sobrepeso & 18 & $31,0(0,96-52,3)$ & 13 & $22,4(0,26-45,0)$ \\
\hline Obesidade & 7 & $12,1(0,12-14,2)$ & 8 & $13,8(0,10-27,7)$ \\
\hline
\end{tabular}

A frequência de resultados nas diferentes categorias do desenvolvimento motor está apresentada na FIGURA 1 , sendo possível verificar que houve um aumento significativo $(\mathrm{p}<0,001)$ de alunos na zona verde em todos os domínios avaliados, o que evidencia que estão no seu desenvolvimento típico do desempenho motor. É possível identificar ainda que, após o experimento em todas as categorias, a frequência de alunos na zona vermelha (Desordem de Coordenação de Desenvolvimento) diminuiu mais da metade quando comparado com o pré-teste.

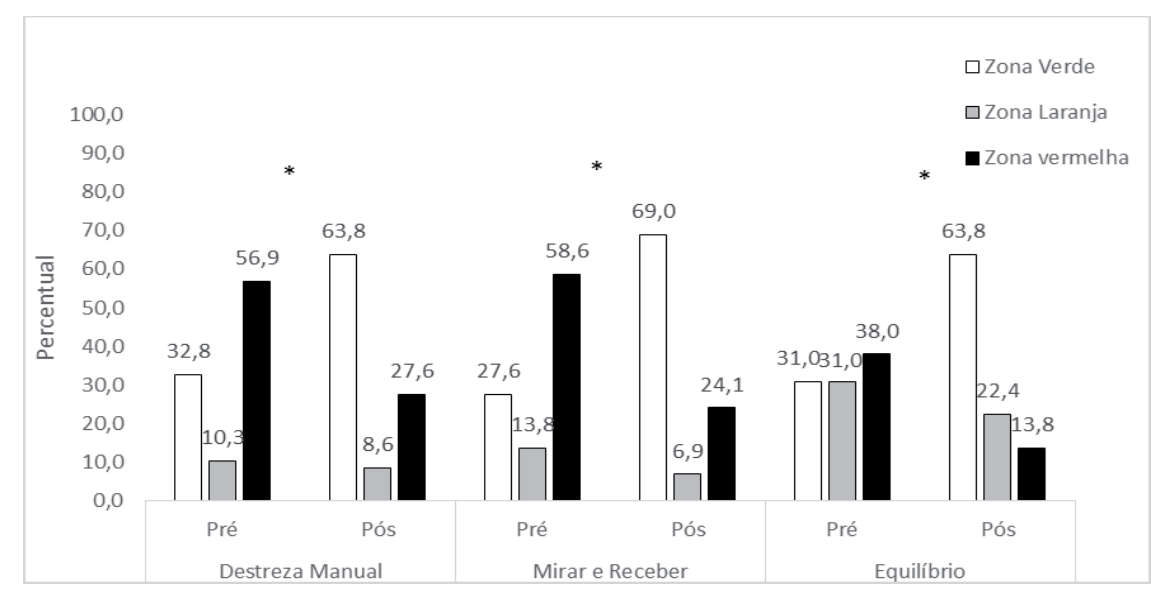

FIGURA 1- Distribuição de frequência das diferentes categorias do Desenvolvimento Motor de acordo com os testes, Uruguaiana, 2015. 
A TABELA 2 apresenta os valores de mediana e intervalo interquartil, bem como, as diferenças entre as duas testagens para as habilidades motoras destreza motora, mirar e receber, equilíbrio, escore total e IMC, sendo que os resultados apresentaram diferença significativa quando comparados os valores de pré-teste e o pós-teste, mostrando que os resultados foram positivos após a intervenção motora proposta, observada pelo aumento da mediana. Além disso, o tamanho do efeito da intervenção para estas variáveis foi moderado. Vale ressaltar que o índice de massa corporal dos avaliados não apresentou diferenças significativas entre o pré e o pós teste ( $>0,05)$.

TABELA 2 - Valores descritivos e inferenciais dos escores das categorias e total do teste de Desenvolvimento motor pré e pós-teste, Uruguaiana, 2015.

\begin{tabular}{|c|c|c|c|c|c|c|c|c|}
\hline \multirow{2}{*}{ Variáveis } & \multicolumn{2}{|c|}{ Pré } & \multicolumn{2}{|c|}{ Pós } & \multirow{2}{*}{$\mathbf{p}$} & \multirow{2}{*}{ TE } & \multirow{2}{*}{$\mathrm{MMD}_{95}$} & \multirow{2}{*}{ N (\%) } \\
\hline & Mediana & $\mathrm{IQ}(25-75)$ & Mediana & $\operatorname{IQ}(25-75)$ & & & & \\
\hline $\begin{array}{l}\text { Destreza Motora } \\
\text { (pontos) }\end{array}$ & 17,0 & $13,0-22,0$ & 24,0 & $17,7-29,0$ & $<0,001^{*}$ & 0,403 & 1,36 & $42(72,4)$ \\
\hline $\begin{array}{l}\text { Mirar e Receber } \\
\text { (pontos) }\end{array}$ & 11,5 & $9,7-15,0$ & 15,5 & $12,7-19,0$ & $<0,001^{*}$ & 0,404 & 0,86 & $44(75,9)$ \\
\hline Equilíbrio (pontos) & 20,0 & $16,7-24,2$ & 25,5 & $20,7-30,0$ & $<0,001^{*}$ & 0,376 & 1,56 & $41(70,7)$ \\
\hline Escore Total (pontos) & 49,0 & $43,0-58,0$ & 68,5 & $55,7-75,2$ & $<0,001^{*}$ & 0,505 & 2,18 & $51(87,9)$ \\
\hline IMC (kg/m2) & 16,6 & $15,7-18,7$ & 16,8 & $15,5-18,3$ & 0,704 & 0,035 & 0,40 & $18(31,3)$ \\
\hline
\end{tabular}

A mínima mudança detectável $\left(\mathrm{MMD}_{95}\right)$ também está apresentada na TABELA 2, indicando que, de acordo com os dados obtidos, um valor de mudança observado em um aluno numa situação de pós-intervenção que apresente variação menor à indicada na Tabela, não pode ser distinguível do erro de medida, evidenciando que não houve qualquer alteração na variável. Desta forma, para considerar que houve alteração significativa oriunda da intervenção, o avaliado deve apresentar uma mudança mínima de 1,36 pontos da Destreza motora, 0,86 pontos no Mirar e receber, 1,56 pontos no equilíbrio e 2,18 pontos no escore total. Com base nestes resultados, foi possível identificar que a maioria dos avaliados atingiu a mínima mudança detectável $\left(\mathrm{MMD}_{95}\right)$ nas variáveis do desenvolvimento motor após a intervenção, o que não foi observado no Índice de Massa Corporal (31,3\%).

A análise da relação do desenvolvimento motor com o sexo está apresentada na TABELA 3 , sendo possível identificar que existe diferenças significativas entre os sexos, apenas no pósteste da categoria "Mirar e receber" $(\mathrm{p}=0,008)$. Quando analisada a diferenças entre o pré e o pós teste por grupos, ambos os sexos apresentaram diferenças significativas em todas as categorias do desenvolvimento motor, com tamanho de efeito moderado, o que não foi evidenciado no estado nutricional $(p>0,05)$.
$\mathrm{IQ}=$ intervalointerquartil, p= significância, * valor significativo no teste de Wilcoxon; $\mathrm{TE}=$ tamanho do efeito "d" Teste de Cohen; MMD95= Mínima mudança detectável; $N(\%)=$ frequência absoluta e relativa dos indivíduos que atingiram a mínima mudança detectável. 
TABELA 3 - Valores do pré-teste e pós-teste de cada habilidade e associação entre os sexos Unipampa,2015.

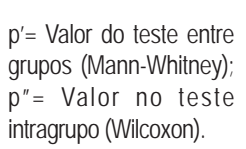

\begin{tabular}{|c|c|c|c|c|c|c|c|c|}
\hline \multirow[b]{2}{*}{ Variáveis } & \multicolumn{2}{|c|}{ Pré-teste } & \multicolumn{4}{|c|}{ Pós-teste } & \multirow{2}{*}{$\begin{array}{c}\text { Masculino } \\
\text { p" }\end{array}$} & \multirow{2}{*}{$\begin{array}{c}\text { Feminino } \\
\text { p" }\end{array}$} \\
\hline & $\begin{array}{l}\text { Masculino } \\
\text { n (\%) }\end{array}$ & $\begin{array}{c}\text { Feminino } \\
\text { n (\%) }\end{array}$ & $\mathbf{p}^{\prime}$ & $\begin{array}{c}\text { Masculino } \\
\mathrm{n}(\%)\end{array}$ & $\begin{array}{c}\text { Feminino } \\
\mathrm{n}(\%)\end{array}$ & $\mathbf{p}^{\prime}$ & & \\
\hline
\end{tabular}

\section{Destreza Motora}

Verde

Laranja

Vermelha

$8(30,8)$

$4(15,4)$

$11(34,4)$

$2(6,2)$

$14(53,8)$

$19(59,4)$

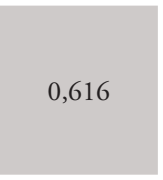

\begin{tabular}{|c|c|c|}
\hline $16(61,5)$ & $21(65,5)$ & \\
\hline $3(11,5)$ & $2(6,4)$ & 0,381 \\
\hline $7(27,0)$ & $9(28,1)$ & \\
\hline
\end{tabular}

$<0,001$

$<0,001$

Mirar e Receber

Verde

$$
10(38,4)
$$

$5(19,2)$

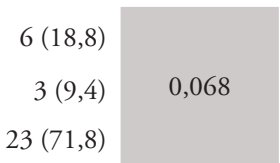

$21(80,8)$

$3(11,5)$

$19(59,4)$

$2(7,7)$

$1(3,2)$

$12(38,4)$

$0,008^{*}$

$<0,001$

$<0,001$

\section{Equilíbrio

Verde
Laranja
Vermelha

$8(30,8)$
$8(30,8)$
$10(38,4)$

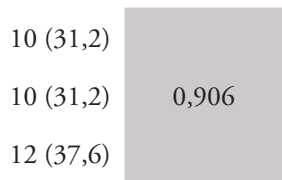
$14(53,8)$
$7(27,0)$
$5(19,2)$

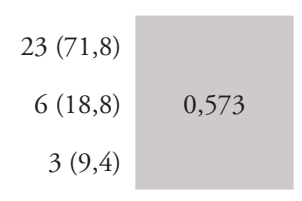

0,004

0,001

\section{Total}

Verde

\begin{tabular}{rr|r}
$1(3,8)$ & $0(0,00)$ & \\
$6(23,1)$ & $12(37,5)$ & 0,857 \\
$19(73,1)$ & $20(62,5)$ & \\
\hline
\end{tabular}
$13(50,0)$
$5(19,2)$

$17(53,1)$

$6(18,8)$

0,944

$<0,001$

$<0,001$

$8(30,8) \quad 9(28,1)$

IMC

$\begin{array}{lcc}\text { Normal } & 15(57,7) & 16(61,6) \\ \text { Sobrepeso } & 7(26,9) & 5(19,2) \\ \text { Obesidade } & 4(15,4) & 5(19,2)\end{array}$

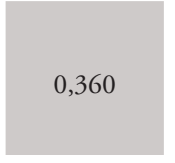

$18(56,2)$

$11(34,4)$

$3(9,4)$
$21(65,6)$
$8(25,0)$

$3(9,4)$

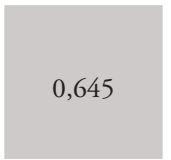

0,909

0,519

\section{Discussão}

Apesar dos estudos com o método Pilates elucidarem efeitos positivos sobre o condicionamento físico, flexibilidade, coordenação motora ${ }^{14}$, força e postura ${ }^{15}$, as evidências científicas que comprovam os benefícios deste método voltados à população pediátrica são limitadas ${ }^{16}$. Dessa forma, nos resultados obtidos pelo presente estudo, observa-se que a intervenção do Método Pilates nas crianças resultou em uma melhora significativa nas três categorias analisadas (destreza motora, mirar e receber e equilíbrio), favorecendo, de forma integral, o desenvolvimento motor desses alunos.
Corroborando com os dados de nosso estudo, o trabalho de Montanez e Lara ${ }^{10}$ evidenciaram melhora significativa sobre o equilíbrio corporal, esquema corporal e organização espacial em crianças, após a prática do método Pilates, identificando efeitos positivos do método sobre os domínios de desenvolvimento motor. Outros estudos mostram uma influência positiva do método Pilates sobre o perfil postural de crianças ${ }^{16,17}$. De fato, Goulart, TeIXeIra e LARA ${ }^{17}$ analisaram o perfil postural de crianças praticantes e não praticantes do método Pilates, e identificaram um melhor alinhamento corporal e do 
centro de gravidade naquelas praticantes do método. De forma semelhante, Silva et al. ${ }^{16}$ destacaram também os benefícios da prática do método sobre o alinhamento postural em uma amostra de crianças.

Os efeitos sobre o alinhamento postural e centro de gravidade podem ser explicitados pelo fato de que o método envolve contraçôes isotônicas e isométricas, com ênfase no powerhouse ou centro de força, o qual é composto pelos músculos abdominais, transverso abdominal, multífidos, assoalho pélvico, glúteos e paravertebrais lombares ${ }^{18}$. Esse centro auxilia a localizar nosso centro de gravidade, estabilizar a coluna e a pélvis durante os movimentos, manter um adequado alinhamento da coluna contra a ação da gravidade, propiciando uma base de suporte para os movimentos dos membros ${ }^{19}$. Dessa forma, considerando a relação entre o centro de forças, postura corporal, centro de gravidade e equilíbrio, pressupóe-se que o método Pilates seja capaz de melhorar o alinhamento postural e a coordenaçáo motora, promovendo melhorias nas habilidades motoras em crianças, fato esse encontrado no presente estudo.

De acordo com AlmeIDA ${ }^{20}$, as experiências durante a infância são necessárias para se alcançar a precisão da maturação cerebral e função neural, e segundo LORENZIN $^{21}$, formarão a base para o aprendizado tanto de habilidades mentais como sociais da criança. Sendo assim, considerando a importância da realização de intervençôes motoras sobre o desenvolvimento integral da criança, alguns estudos ${ }^{22,23,24}$ identificaram melhorias dessas intervençóes sobre o desenvolvimento motor de alunos dos anos iniciais do Ensino Fundamental. BRAGA et al. ${ }^{22}$ identificaram resultados positivos sobre o desenvolvimento motor de 60 crianças, aplicando um protocolo Test of Gross Motor Developemed - 2 (TGMD-2) de intervenção motora, ao longo de 12 semanas (com frequência de 3 vezes na semana). $\mathrm{O}$ estudo de Souza ${ }^{23}$ encontrou efeitos positivos sobre o desenvolvimento motor de 26 meninas, através de uma proposta de intervençáo motora com a dança, ao longo de 10 semanas (com frequência de duas vezes por semana). Adicionalmente, também foi verificado melhora nos resultados do desenvolvimento motor de alunos, por meio de um programa de intervenção motora com características da Educação Física desenvolvimentista, incluindo atividades voltadas para a melhora do desenvolvimento motor amplo, destreza motora e manuseio de objetos ${ }^{24}$.

É possivel verificar que os alunos recentemente ingressaram na escola, sendo que a grande maioria não possuía experiência com nenhuma prática de atividade física orientada, e isto pode demonstrar o motivo, pelo qual, os alunos apresentaram baixos índices de desenvolvimento motor no pré-teste. Adicionalmente, por se tratar de crianças em região de vulnerabilidade social, uma vez que a escola localiza-se em na periferia da cidade, cabe ressaltar que existem fatores que podem influenciar negativamente sobre o desenvolvimento motor dessas crianças, conforme apontam SANTOS et $\mathrm{al}^{25}$. Esses autores reforçam que problemas de ordem familiar, baixo nível socioeconômico, problemas nutricionais, falta de higiene, violência e drogadição influenciam de forma mais expressiva sobre as dificuldades de aprendizagem e as habilidades motoras na infância.

Com base em tais dificuldades é possível fazer algumas analogias com outros esportes, pois o Pilates é uma prática corporal constituída por vários componentes da cultura já existentes, como dança, yoga, esqui, ginástica entre outros ${ }^{26,27}$. Nesse sentido, alguns autores demonstram que, crianças praticantes de ginástica, apresentam uma melhora significativa no desenvolvimento motor. Dessa forma, o estudo de $P_{A Z}{ }^{28}$ mostrou que alunos praticantes de ginástica artística têm melhor desempenho motor do que crianças que apenas fazem as aulas de Educaçáo Física escolar, sendo que os resultados sáo positivos, tanto na locomoção, quanto no manuseio de objetos.

De forma semelhante, AzEVEDO ${ }^{29}$ reitera que crianças praticantes de ginástica rítmica possuem melhores resultados nas habilidades motoras locomotivas e no controle de objeto. Sendo assim, a ginástica artística tem sido considerada indispensável para a ampliaçáo do acervo motor dos alunos ${ }^{30}$.

Em relação ao desenvolvimento motor e a diferença entre o sexo das crianças, o presente estudo encontrou diferença estatística entre meninos e meninas apenas no pós-teste da habilidade mirar e receber. Corroborando, outro estudo também encontrou diferenças entre os sexos, somente na variável de manuseio de objetos, onde os meninos possuíram melhores resultados ${ }^{31}$. Tal diferença pode ser explicada pelo fato dos mesmos apresentarem melhores habilidades em atividades de vigor, por ter maiores experiências motoras ${ }^{32}$, justificada pelo fato de as brincadeiras praticadas por estes serem de maior mobilidade e agilidade.

O estudo não demonstrou relação entre o desempenho motor e o estado nutricional de crianças, e esse resultado vêm ao encontro de outros estudos $33,34,35$, desta maneira, é possível observar que o desenvolvimento motor das crianças independe do seu estado nutricional, reforçando que crianças com sobrepeso e obesidade podem ter um bom 
desenvolvimento motor.

O presente estudo apresenta algumas limitaçóes, pois apesar da melhora significativa obtida nos exercícios é importante relatar que o estudo não contou com um grupo controle, desta forma, não levando em consideração o ganho que eles tiveram no seu desenvolvimento fisiológico e suas experiências motoras adquiridas durante este período (tarefaambiente). Além disso, é necessário destacar a perda amostral, na qual os alunos, por motivos diversos, não obtiveram a frequência mínima de $75 \%$ nas sessóes, sendo excluídos das análises do estudo. Desta forma, como o objetivo inicial do estudo propunha avaliar o total de sujeitos, não foi possível prever um percentual para possíveis perdas. Sendo assim, mesmo a amostra sendo composta por $52,7 \%$ dos indivíduos que o estudo se propôs a avaliar, é necessário avaliar os resultados com cautela, pois de acordo com Miot ${ }^{36}$, quando ocorre uma perda maior de $30 \%$ pode haver comprometimento da representatividade, independente da suficiência numérica dos casos.

Entretanto, com base nos resultados encontrados, foi possível afirmar que a prática do Método Pilates melhorou o desenvolvimento motor das crianças, tendo evidências científicas sobre a importância das práticas de exercício físico para a melhora no desenvolvimento das habilidades motoras nos anos iniciais do ensino fundamental. É observado também que não houve associação entre o estado nutricional e o desenvolvimento motor, sugerindo que o índice de massa corporal não interferiu no desenvolvimento de habilidades motoras, e não foi significativa a comparação entre os sexos.

Sendo assim, é necessário que outros estudos sejam feitos com o Método Pilates na população infantil, a fim de que seja possível elucidar melhor os seus benefícios.

\begin{abstract}
The influence of the Pilates method in motor development of children: a intervention study

The motor development consists of a continuous change of motor behavior throughout life, caused by the interaction between the requirement of the motor task, the individual biology and environmental conditions. The aim of this study was to analyze the influence of Pilates on the children of motor development and whether is associated with the nutritional status and sex of children. The sample consisted of 58 students with seven years old and $55.2 \%$ women. The study was characterized as quasi-experimental, consisting of pre-test and post-test and an experimental treatment with Pilates. The Motor Development was evaluated by means of the Movement Assessment Battery Test for Children (MABC), divided in three categories: manual dexterity, skills with ball and balance tests. To analyze the nutritional status of students was used a digital scale and a stadiometer. The results showed that motor development had a significant improvement after the application of the Pilates method. There was no statistical difference between the sexes as well, there was no association between nutritional status and motor skills development. Based on the results it is possible to conclude that the Pilates Method can contribute to the motor development of children.
\end{abstract}

KeYwords: Child Development; Postural Balance; Motor Activity; Child.

\title{
Referências
}

1. Gallahue DL, Ozmun JC, Goodway JD. Compreendendo o desenvolvimento motor: bebês, crianças, adolescentes e adultos. Porto Alegre: Artmed; 2013.

2. Koutra K, Chatzi L, Roumeliotaki T, et al. Sociodemographic determinants of infant neurodevelopment at 18 months of age: Mother-Child Cohort (Rhea Study) in Crete, Greece. Infant Behav Dev. 2012;35:48-59.

3. Nazario PF, Vieira JLL. Sport context and the motor development of children. Rev Bras Cineantropom Desempenho Hum. 2014;16:86-95.

4. Rosa Neto F, Almeida GMF, Caon G, Ribeiro J, Caram JA, Piucco EC. Desenvolvimento Motor de crianças com 
indicadores de dificuldades na aprendizagem escolar. Rev Bras Ci Mov. 2007;15:45-51.

5. Brasil. Ministério da Educação e Desporto. Secretaria de Ensino Fundamental. Parâmetros Curriculares Nacionais. Brasília: MEC/ SEF, 1997a (Área: Educação Física; Ciclos: 1 e 2).

6. Jago R, McMurray RG, Bassin S, Pyle L et al. Modifying Middle School Physical Education: Piloting Strategies to Increase Physical Activity Pediatr Exerc Sci 2009;21(2):171-185.

7. Santos GO, Rebouças GM, Filho NJB de A et al. Competência profissional: aulas de Educação Física Escolar nas séries iniciais. EFDeportes.com [Revista Digital]. 178(17). Disponível em http://www.efdeportes.com [2015jun 12]; 2013

8. Pilates JH. The complete writings of Joseph H. Pilates: Return to life through contrology and your health. Philadelphia: Bain Bridge Books, 2000.

9. Pereira CM, Júnior AJC, Campos RS. Efeitos do método pilates na dor lombar - Revisão de literatura. Rev Eletr Ciência. 2013;3(1):23-37.

10. Montanez DR, Lara S. A influência do método Pilates sobre o desenvolvimento motor de crianças. Rev Bras Ci Mov. 2015;23(4):64-71.

11. Hendersonh SE, Sugden DA. Movement Assessment Battery for Children - MABC. London: Psychological Corporation; 1992.

12. Valentini NC, Ramalho MH, Oliveira MA. Movement assessment battery for children-2: translation, reliability, and validity for Brazilian children. Research Develop Mental Dis. 2014; 35(3):733-740.

13. Haley SM, Fragala-Pinkham MA. Interpreting change scores of tests and measures used in physical therapy. Physical Therapy. 2006; 86, 735-743

14. Comunello JF. Benefícios do método pilates e sua aplicação na reabilitação. Instituto Salus:1-12; 2011.

15. Segal NA, Hein J, Basford JR. The effects of Pilates training on flexibility and body composition: an observational study. Arch Phys Med Rehab. 2004;85(1):1977-81.

16. Silva VS, Mascarenhas JC, Sá de A.M, Sá de C.K.C. Efeito de um programa de exercícios baseado no método Pilates sobre a postura de crianças: um estudo piloto. Rev Bras Fisiot. 2015;16(2):152-157.

17. Goulart IP, Teixeira LP, Lara S. Postural analysis of cervical spine and shoulder girdle of children practitioners and non-practitioners of the Pilates method. Fisioter Pesq.; 2016;23(1):38-44.

18. Sinzato CR, Taciro C, Pio CA, Toledo AM, Cardoso JR, Carregaro RL. Efeitos de 20 sessóes do método Pilates no alinhamento postural e flexibilidade de mulheres jovens: estudo piloto. Fisiot Pesq. 2013; 20(2):143-150.

19. O’Sullivan P. Lumbar segmental 'instability': clinical presentation and specific stabilizing exercise management. Man Ther. 2000; 5(1):2-12.

20. Almeida CS. Intervenção motora: efeitos no comportamento do bebê no terceiro trimestre de vida em creches de Porto Alegre (Dissertação). Porto Alegre: Universidade Federal do Rio Grande do Sul, 2004, 199 p.

21. Lorenzini MV. Brincando a brincadeira com a criança deficiente: novos rumos terapêuticos. São Paulo: Manole; 2002

22. Braga RK, Krebs RJ, Valentini NC, Tkac CM. A influência de um programa de intervenção motora no desempenho das habilidades locomotoras de crianças com idade entre 6 e 7 anos. Rev Educ Fis. 2009;20:171-81.

23. Souza MC, Berleze A, Valentini NC. Efeitos de um programa de educação pelo esporte no domínio das habilidades motoras fundamentais e especializadas: ênfase na dança. Rev Educ Fis. 2008;19:509-19.

24. Silva EVA, Contreira AR, Beltrame TS, Sperandio FF. Programa de intervenção motora para escolares com indicativo de transtorno do desenvolvimento da coordenação - TDC. Rev Bras Ed Esp. 2001;17:137-50.

25. Santos CR, Silva CC, Damasceno ML, Medina-Papst J, Marques. Efeito da atividade esportiva sistematizada sobre o desenvolvimento motor de crianças de sete a 10 anos. Rev Bras Educ Fís Esporte. 2015;29(3):497-506.

26. Robinson L, Napper H. Exercícios inteligentes com Pilates e Yoga. São Paulo: Pensamento; 2002.

27. Latey P. The Pilates method: history and philosophy. J Bodywork Mov Ther. 2001;5:275-82

28. Paz B, Pires V. Desempenho motor de crianças praticantes e não praticantes de ginástica rítmica (Trabalho em Congresso). Curitiba: Pontifícia Universidade Católica do Paraná, 2011.

29. Azevedo KA, Palma MS. Desempenho motor de crianças praticantes e não praticantes de ginástica rítmica (Monografia). Porto Alegre: Universidade Federal do Rio Grande do Sul (UFGRS), 2002.

30. Bezerra SP, Ferreira Filho RA, Feliciano JG. A importância da aplicação de conteúdos da ginástica artística nas aulas de educação física no ensino fundamental de $1^{\mathrm{a}} \mathrm{a} 4^{\mathrm{a}}$ série. Rev Mackenzie Educ Fís Esporte. 2006; 5:127-34

31. Brauner LM, Valentini NC. Análise do desempenho motor de crianças participantes de um programa de atividades físicas. Rev Educ Fis 2009;20:5-16.

32. Valentini NC. Percepçôes de competência e desenvolvimento motor de meninos e meninas: um estudo transversal. 
Movimento. 2002;8:51-62.

33. Catenassi FZ, Marques I, Bastos CB, Basso L, Ronque ERV, Gerage AM. Relação entre índice de massa corporal e habilidade motora grossa em crianças de quatro a seis anos. Rev Bras Med Esporte. 2007;13:227-30.

34. Machado HS, de Campos W, da Silva, S.G. Relação entre composição corporal e a performance de padrôes motores fundamentais em escolares. Ativ Fís \& Saúde. 2002;7:63-70.

35. Miranda TB, Beltrame TS, Cardoso FL. Desempenho motor e estado nutricional de escolares com e sem transtorno do desenvolvimento da coordenação. Rev Bras Cineantropom Des Hum. 2001;13:59-66.

36. Miot HA. Tamanho da amostra em estudos clínicos e experimentais. J Vasc Bras. 2011;10 (4):275-278.

\begin{tabular}{r|l} 
ENDEREÇO & Submetido: 01/02/2017 \\
Simone Lara & 1a revisão: 06/09/2017 \\
BR 472, KM 592 & 2a revisão: 11/01/2018 \\
na - RS- BRASIL & 3a revisão: 14/07/2018 \\
@unipampa.edu.br & Aceito: 08/08/2018
\end{tabular}

258 • Rev Bras Educ Fís Esporte, (São Paulo) 2020 Abr-J un;34(2):249-58 